\title{
Pode o conceito de vulnerabilidade apoiar a construção do conhecimento em Saúde Coletiva?
}

\author{
Can the vulnerability concept support the construction \\ of knowledge in collective health care?
}

Alba Idaly M uñoz Sánchez ${ }^{1}$

Maria Rita Bertolozzi 2

Abstract The term vulnerability has been frequently used in scientific literature, especially after the 80's, in some epidemiologic studies, mainly focusing therisk perspective. The purpose of this article is to review the vulnerability concept through a systematic study of national and international circulation newspapers, to support knowledge and practices in the collective health care field. Furthermore, it indicates how this concept has been revisited in studies conducted in Brazil, mentioning issues which support thestructural dimension of reality, linking them to the needs of a program for patients, health care users and their families. It is important to study the vulnerability concept due to the fact that an understanding of this concept will contribute to support the delivery of care, of collective health care including individual health care programs and health care policies.

Key words Vulnerability, Publichealth, Risk factors
Resumo 0 termo vulnerabilidade tem sido usado freqüentemente na literatura científica, especialmente após a década de 80, em vários estudos epidemiológicos, principalmente focalizado para a perspectiva de risco. Esteartigo tem por objetivo apresentar uma revisão sobre o conceito devulnerabilidade, a partir de um estudo sistemático em periódi cos de circulação nacional einternacional, para subsidiar o conhecimento ea prática da Saúde Coletiva. A presenta, ainda, como no Brasil esse conceito tem sido revisitado, incorporando questões que sustentam a dimensão estrutural da realidade, articulando-a àsnecessidadesque emanam do plano singular, que se refere aos usuários de saúde/pacientes e suas respectivas famílias. A importância de se estudar o conceito de vulnerabilidade resi de no fato de que pode embasar as práticas de saúde, neste caso de Saúde Coletiva, integrando os planos relativosaosindivíduos, aosprogramas e às políticas de saúde.

Palavras-chave Vulnerabilidade, Saúde coletiva, Fatores de risco 
Aproximações ao conceito de vulnerabilidade

0 termo vulnerabilidade tem sido bastante empregado nos últimos anos, expressando distintas perspectivas de interpretação. Wisner ${ }^{1}$ analisa 0 conceito sob a perspectiva da vulnerabilidadeque as pessoas/populações podem apresentar em caso de exposição a acidentes extensivos, como terremotos. 0 autor aponta que a vulnerabilidade ea capacidade são lados deum mesmo processo, pois a primeira está intimamente relacionada à capacidade de luta e de recuperação que o indivíduo pode apresentar. Argumenta, ainda, que o nível socioeconômico, a ocupação e a nacionalidade também se relacionam a esse processo, pois repercutem sobre 0 acesso à informação, aos serviços e à disponibilidade de recursos para a recuperação, os quais, por sua vez, potencializam ou diminuem a vulnerabilidade.

Dilley eBoudreau ${ }^{2}$ empregam o termo na área de nutrição, definindo-o como a possibilidade de sofrer danos e a capacidade para o seu enfrentamento. Nessa abordagem, defende-se a necessidade de se identificar: quem, por que e em que nível os grupos são vulneráveis.

Numa outra perspectiva, Kalipeni ${ }^{3}$ aponta que Watts e Bohle, em 1993, propuseram uma estrutura tripartite para constituir uma teoria sobre a vulnerabilidade, que consiste em entitlement, empowerment e política econômica. A vulnerabilidade é definida na intersecção desses três poderes, sendo que entitlement refere-se ao direito das pessoas; empowerment, o empoderamento, que se refere à sua participação política e institucional; e a política econômica, se refere à organização estrutural-histórica da sociedade e suas decorrências. Desse modo, a vulnerabilidade às doenças e situações adversas da vida distribui-se de maneira diferente segundo os indivíduos, regiões e grupos sociais e relaciona-se com a pobreza, com as crises econômicas e com o nível educacional. Ao multifatorializar a vulnerabilidade, acrescenta, ainda, que a vulnerabilidade depende do local edo clima, restringindo-se, portanto, à dimensão geográfica ${ }^{3}$. Especificamentena área da saúde, o quadro analítico da vulnerabilidadeemerge no começo da década 80, como possibilidade de interpretação à epidemia da AIDS, na perspectiva de reconceituar a tendência individualizante da doença. Assim, Delor e Hubert ${ }^{4}$ apresentam uma matriz heurística, construída a partir de pesquisa realizada com pessoas portadoras do HIV/AIDS, na Bélgica, em 1997. Os resultados da investigação são analisados sob três dimensões: trajetória social, interação e contexto social. A trajetória social refere-se às diferentes etapas que as pessoas atravessam no curso da vida, assim como as condutas assumidas nesse curso. A dimensão relativa à interação refere-seà relação entre os indivíduos e o contexto social e incorpora os fatores econômicos, políticos eculturais, numa dada sociedade, assim como as várias maneiras de atuação e de relação entre os indivíduos. Esses níveis podem ser analisados desdedoisângulos, objetiva esubjetivamente (o que os autores denominam como dimensão sóciosimbólica). A análise final que se apresenta propõe a construção de processos de identidade e de vulnerabilidade dos indivíduos estudados.

Nessa mesma linha, Mann et al. ${ }^{5}$, no livro "Aids no mundo", apresentam uma metodologia para avaliar a vulnerabilidadeà infecção pelo HIV e AIDS. Segundo os autores, o comportamento individual é o determinante final da vulnerabilidadeà infecção, o quejustifica focalizar ações no indivíduo, embora isto não seja suficiente para o controle da epidemia. Deste modo, é importante considerar outros fatores que podem influenciar tal controleno âmbito individual. Propõem uma estrutura de análise que incorpora o comportamento individual, o âmbito coletivo eo social. 0 primeiro refere-se à auto-avaliação, através de perguntas, para se verificar o conhecimento, os comportamentos, 0 status social e 0 acesso à informação, avaliando-se como pode ocorrer a transmissão da doença. A vulnerabilidade coletiva refere-se à avaliação da capacidade estrutural e funcional dos programas de controle da epidemia ea vulnerabilidade social consiste na avaliação das realidades sociais através de indicadores do Programa de Desenvolvimento das $N$ ações Unidas. Finalmente, os autores propõem a aplicação de um sistema de escores, que classificam a vulnerabilidade como alta, média e baixa. Nessa perspectiva, enfatizam a necessidade de que os indivíduos se responsabilizem pela prevenção da doença, imputando aos mesmos essa tarefa.

Segundo Paris ${ }^{6}$, o modelo original, proposto por $\mathrm{M}$ ann et al. ${ }^{5}$, apresenta-se impregnado da tradição norte-americana de pesquisa social, ao focalizar o âmbito individual segundo aspectos comportamentais e cognitivos. Paris ${ }^{6}$ apresenta um estudo sobre vulnerabilidade à Aids, numa perspectiva psicossocial, a partir da população de mulheres acometidas. A presenta uma decodificação do conceito em: vulnerabilidade relacionada aos fatores estruturais da sociedade, que se refere à desigualdade de renda, educação e de acesso à serviços; vulnerabilidade relacionada aos aspec- 
tos fisiológicos e decorrentes das relações de gênero na sociedade e a vulnerabilidade na esfera da significação, que integra o conceito de risco e a maneira como se expressa, no imaginário social, além de seus diferentes significados através da história.

$\mathrm{Na}$ tentativa de ampliar o conceito proposto por Mann et al. ${ }^{5}$, Ayres et al. ${ }^{7}$, no Brasil, apontam que o modelo de vulnerabilidade está conformado por três planos interdependentes de determinação e, conseqüentemente, de apreensão da maior ou da menor vulnerabilidade do indivíduo e da coletividade. 0 ol har do autor busca a compreensão do comportamento pessoal ou a vulnerabilidadeindividual, do contexto social ou vulnerabilidade social e do programa de combateà doença, no caso a AIDS, ou vulnerabilidadeprogramática.

0 significado do termo vulnerabilidade, nesse caso, refere-se à chance de exposição das pessoas ao adoecimento, como resultante de um conjunto de aspectos que ainda que se refiram imediatamente ao indivíduo, o recoloca na perspectiva da dupla-face, ou seja, o indivíduo e sua relação com o coletivo. Explicando melhor, o indivíduo não prescinde do coletivo: há relação intrínseca entre os mesmos. Além disso, o autor propõe que a interpretação da vulnerabilidade incorpore, necessariamente, o contexto como lócus devulnerabilidade, o que podeacarretar maior suscetibilidadeà infecção e ao adoecimento e, demodo inseparável, à maior ou menor disponibilidade de recursos de todas as ordens para a proteção das pessoas contra as enfermidades ${ }^{7}$.

0 marco conceitual que propõe 0 autor diferedo marco inicial proposto por $\mathrm{M}$ ann et al. ${ }^{5}$, pois Ayres et al. ${ }^{7}$ não enfatiza excessivamentea vulnerabilidade à determinação individual. Para esse autor, a unidade analítica está constituída no indivíduo-coletivo. Nessa perspectiva, propõe a sua operacionalização através da Vulnerabilidade Individual, que se refere ao grau e à qualidade da informação que os indivíduos dispõem sobre os problemas de saúde, sua elaboração e aplicação na prática; a Vulnerabilidade Social, que avalia a obtenção das informações, o acesso aos meios de comunicação, a disponibilidade de recursos cognitivos emateriais, o poder de participar de decisões políticas e em instituições; e a VulnerabilidadeProgramática, que consistena avaliação dos programas para responder ao controle de enfermidades, além do grau e qualidade de compromisso das instituições, dos recursos, da gerência edo monitoramento dos programas nos diferentes níveis de atenção?.
Cada um desses planos podeser tomado como referência para interpretar-se também outros agravos, além da AIDS. Essa abordagem pode ampliar a atuação em saúde e gerar reflexões que podem ser úteis para a formulação de políticas de saúdea partir das necessidades da coletividade.

Nesse sentido, o modelo propõe construir políticas voltadas às necessidades dos seres humanos, trabalhar com as comunidades e realizar diagnósticos sobre as condições dos grupos sociais, de maneira participativa, assi $m$ como a redefinição dos objetos deintervenção ea análise crítica das práticas de saúde para a sua reconstrução orientada às necessidades dos indivíduos eda coletividade.

Entende-se, portanto, a partir dessa última perspectiva apresentada, o conceito de vulnerabilidade como um convite para renovar as práticas de saúde como práticas sociais e históricas, através do trabal ho com diferentes setores da sociedade e da transdisciplinaridade. Isso permite - repensar sobre as práticas, de maneira crítica e dinâmica, para contribuir na busca de mudanças políticas, culturais, cognitivas etecnológicas, que promovam impacto nos perfis epidemiológicos ${ }^{8}$.

Este último conceito de vulnerabilidade traz implícita, ainda, a intervenção e exige a adoção de um marco referencial diverso do utilizado historicamente pela Epidemiologia Clássica. Desse modo, a vulnerabilidade não nega o modelo biológico tradicional, muito pelo contrário, o reconhece, mas busca superá-lo. Vale lembrar que 0 modelo privilegia, como unidade analítica, o plano do coletivo, e que a estrutura é marcada por um referencial ético-filosófico, que busca a interpretação crítica dos dados. Essa perspectiva analítica amplia o horizonte para além da abordagem quese restringeà responsabilidadeindividual, que é empregada, tradicionalmente, em vários estudos que analisam o papel da pessoa na trama da causalidade. Ademais, incorpora o trabalho participante com a população, de maneira a contribuir para que esta seja "sujeito de sua vida"9.

Outrosautores também têm feito uso do conceito de vulnerabilidade para a explicação de certos processos, como Palma e M attos ${ }^{10}$, que definem o conceito de vulnerabilidade social, relacionando-0 a processos deexclusão, discriminação ou enfraquecimento dos grupos sociais e sua capacidade de reação. É necessário destacar queessa abordagem é muito similar à proposta conceitual de Ayres, pois envolve aspectos que dizem respeito à dimensão estrutural, quando os autores se reportam aos processos de exclusão social, e relativos às dimensões particular esingular, quan- 
do fazem menção à capacidade de reação dos indivíduos/grupos sociais, parao enfrentamento da doença.

Especificamente em relação à operacional ização do conceito de vulnerabilidade, Figueiredo e Ayres ${ }^{11}$ apresentam um processo de intervenção comunitária para a redução da vulnerabilidade de mulheres às doenças de transmissão sexual, numa favela de São Paulo. Os autores verificaram que as estratégias que respondiam às necessidades e interesses da comunidade, além daquelas que respondiam às representações sobre essas enfermidades, tiveram sucesso, o que mostra a importância de se aproximar à população e conhecer suas necessidades, já que é a partir dela quese podem encontrar alternativas deintervenção às vulnerabilidades.

Com o intuito de analisar o curso da epidemia da AIDS, nos últimos dez anos, Paiva et al. ${ }^{9}$ discutem a necessidade de superação das noções de "grupo derisco" e de "comportamentos derisco" em relação à doença. Os autores mostram as importantes contribuições que os trabalhos, sob a ótica da vulnerabilidade, têm aportado em relação à educação eà saúde. Alguns destaques que os autores apontam são o trabal ho junto à população, fomentando a solidariedade, através deprocesso mútuo de aprendizagem entre os profissionais de saúde e a população.

\section{Um exemplo de operacionalização do conceito de vulnerabilidade}

Partindo-se do conceito de vulnerabilidade proposto por Ayres $^{8}$ e alicerçadas na interpretação da saúde-doença como processo social, Muñoz Sánchez e Bertolozzi ${ }^{12}$ analisaram como se processava a vul nerabilidadeà tuberculose, num grupo de estudantes universitários. Com base em critérios da Sociedade Americana Torácica e do Centro para o Controle de Doenças $(C D C)^{13}$, as autoras construíram uma matriz, utilizando categorias e definindo escores de vulnerabilidade: média ealta. As categorias utilizadas foram: menção à contato com portadores de tuberculose, procedência de países e/ou de regiões do Brasil com taxas de tuberculose maiores que 30, uso de drogas edormir em local com mais detrês pessoas. Essas categorias foram analisadas em conjunto com outras, que se referem à vulnerabilidade individual e social, verificando-se também como fundamentais a situação de empregabilidade dos pais e dos próprios alunos, o acesso à informação, as crenças religiosas, as possibilidades deaces- so ao lazer, dentre outras, que minimizam ou potencializam a vulnerabilidade à tuberculose.

No mesmo estudo, Muñoz Sánches e BertoIozzi ${ }^{12}$ analisaram a Vulnerabilidade Programática, com relação à unidade de saúde que era referência para o grupo de alunos estudados. Verificou-se que a dificuldade de acesso ao serviço de saúde se constituiu como importante marcador de vulnerabilidade.

Algumas questões que podem apoiar as práticas de Saúde Coletiva

0 modelo devulnerabilidade queinterliga os aspectos individuais, sociais e programáticos reconhece a determinação social da doença e se coloca como um convite para renovar as práticas de saúde, como práticas sociais e históricas, envolvendo diferentes setores da sociedade. Assim, é mister superar estudos que restringem as análises à perspectiva da multifatorialidade e ocultam a complexidade das verdadeiras causas da doença. A Teoria da Determinação Social apreende a saúde-doença como uma síntese do conjunto de determinações e que acaba por resultar em riscos ou potencialidades, que se evidenciam em perfis ou padrões saúde-doença. Deste modo, essa Teoria analisa como, em última instância, os fatores estruturais estão vinculados à emergência de processos saúde-doença, diferentes na sua expressão, de acordo com a inserção de classe dos indivíduos ${ }^{14}$.

A abordagem na perspectiva da determinação social da saúde-doença e que o modelo de vulnerabilidade apresentado incorpora ${ }^{12}$ aponta para a necessidade da transdisciplinaridade, o que é fundamental quando se trata de problemas ou de necessidades de saúde, na medida em que a complexidade do objeto da saúde requer diferentes aportes teórico-metodológicos, sob pena de reduzir as ações a "tarefas" pontuais, de caráter emergencial, que não modificam a estrutura da teia de causalidade.

Vale enfatizar que a interpretação da saúdedoença, além de se apoiar nos processos de produção e de reprodução social, não deve descolar-se da dimensão subjetiva, que diz respeito às representações/significados que os indivíduos atribuem a fatos e à vida em si, o que acaba por refletir-se nos comportamentos e atitudes das pessoas.

Assim, a vulnerabilidade deve levar em conta a dimensão relativa ao indivíduo e o local social por ele ocupado. Ao propor outras perspectivas 
de abordagem, como a programática e a social, permite a integralização da análise da situação de saúde e de diferentes possibilidades de intervenção, sempre contemplando a participação dos indivíduos.

Para intervir em situações de vulnerabilidade é imperativo o desenvolvimento de ações que envolvam "resposta social" que, segundo Ayres et al. ${ }^{7}$, diz respeito à participação ativa da população na procura solidária de estratégias passíveis de execução e de encaminhamento/equacionamento de problemas e de necessidades de saúde.

\section{Considerações finais}

Verifica-se que a vulnerabilidade supera o marco do conceito de risco, tradicionalmente empregado no âmbito da Epidemiologia Clássica, pois este, segundo Ayres", designa " [...] chances probabilísticas de suscepti bilidade, atribuíveis a um indivíduo qualquer de grupos populacionais particularizados, delimitados em função da exposibilidade a agentes (agressores ou protetores) de interesse técnico ou científico". A ssim, em saúde têm-se utilizado o conceito de "risco" como instrumento de caráter probabilístico para orientar as atividades de intervenção em saúde, 0 que suscitou a Ayres $^{15}$ alertar sobre a necessidade de revisitar a relação entre risco e intervenções em saúde, salientando que os "objetos" em saúde são sujeitos e apontando nas limitações desse conceito o seu caráter "objetivista" e "analítico-discriminativo".

Já o conceito de Vulnerabilidade, novo, em processo de construção, supera o caráter indivi- dual izantee probabilístico do clássico conceito de "risco", ao apontar a vulnerabilidade como um conjunto de aspectos que vão além do individual, abrangendo aspectos coletivos, contextuais, que levam à suscetibilidade a doenças ou agravos. Esse conceito também leva em conta aspectos que dizem respeito à disponibilidade ou a carência de recursos destinados à proteção das pessoas.

A Saúde Coletiva pode amplamente valer-se dessa última perspectiva apresentada, o que permite um avanço nas alternativas concretas de intervenção. Coloca-se como potencial instrumento para a transformação das práticas de saúde. As possibilidades de leitura das necessidades dos indivíduos, a partir do conceito ampliado devulnerabilidade, coloca à Saúde Coletiva, na medida em que incorpora práticas cuja essência é o cuidado ao indivíduo-coletivo, a possibilidade de apoiar os sujeitos sociais no que diz respeito aos seus direitos, fato que, na atual conjuntura de saúde e de desenvolvimento do país, se constitui como um desafio a ser perseguido e concretizado.

Assim, enfatiza-se que a operacionalização do conceito de vulnerabilidade pode contribuir para renovar as práticas de saúde coletiva, nas quais o cuidado às pessoas deve ser responsabilidade de diferentes setores da sociedade, através da multidisciplinariedade, além de conjugar diversos setores da sociedade, todos querendo e construindo projetos, "cuidando" da população, tendo como base que "[...] a atitude de cuidar não pode ser apenas uma pequena e subordinada tarefa parcelar das práticas de saúde. A atitude "cuidadora" precisa se expandir mesmo para a totalidade das reflexões e intervenções no campo da saúde" ${ }^{15}$.

\section{Colaboradores}

AIM Sánchez procedeu à revisão bibliográfica e redação do artigo. M R Bertolozzi orientou a sua elaboração.

\section{Agradecimentos}

As autoras agradecem ao CNPq pelo apoio no desenvolvimento do estudo. 


\section{Referências}

1. Wisner B. Marginality and vulnerability. Appl Geogr [serial on the Internet]. 1998 Jan [cited 2001 Ago 10]; 18(1):[about 9 p.] Available from: http://www. sciencedirect.com/science

2. Dilley M, Boudreau TE. Coming to terms with vulnerability: a critique of the food security definition. Food Police 2001; 26(3):229-47.

3. Kalipeni E. Health and disease in southern Africa: a comparativeand vulnerability perspective. Soc Sci M ed [serial on the Internet.] . 2000 Apr [cited 2001 N ov 11]; 50(7/8):[about 19 p.]. Available from: http://www. sciencedirect.com/science

4. Delor F, Hubert M . Revisiting the concept vulnerability. Soc Sci M ed [serial on the Internet]. 2000 ]un [cited 2002 Jun 10];50(11):[about 20 p.]. Available from: http://www.sciencedirect.com/science

5. M ann J, Tarantola DJM , N etter T. Como avaliar a vulnerabilidade à infeção pelo HIV e AIDS. In: Parker R. A AIDS no mundo. Rio de Janeiro: Relume Dumará; 1993. p. 276-300.

6. Paris SMJ. Aspectos psicossociais e a vulnerabilidade feminina. Folha M édica UN IFESP 1999; 18(1):41-5.

7. Ayres JRCM, França Junior I, Calazans $G$, Sall letti $H$. Vulnerabilidade e prevenção em tempos de Aids. In: Barbosa R, Parker R, organizadores. Sexualidade pelo avesso: direitos, identidades e poder. Rio de Janeiro: Relume Dumará; 1999. p. 50-71.

8. Ayres JRCM. Sobre o risco: para compreender a epidemiologia. São Paulo: Hucitec; 2002.
9. Paiva V, Peres C, Blessa C. Jovens e adolescentes em tempos de AIDS: reflexões sobre uma década de trabalho de prevenção. Rev Psicol USP [periódico na Internet]. 2002 [acessado 2003 Ago. 12];13(1):[cerca de 20 p.]. Disponível em: http://www. scielo.br/scielo.php?

10. Palma A, M attos UAO. Contribuições da ciência pósnormal à saúde pública e a questão da vulnerabilidade social. Hist Ciênc Saúde M anguinhos [ periódico na Internet]. 2001 Set./D ez. [acessado 2003 Jan 5];8(3):[cerca de 23 p.]. Disponível em: http://www.scielo.br/ scielo.php?

11. Figueiredo R, AyresJRCM . Intervenção comunitária e redução da vulnerabilidade de mulheres às DST/Aids em São Paulo, SP. Rev Saúde Pública 2002; 36(4):1-17.

12. Muñoz Sánchez AI, Bertolozzi M R. Conhecimentos e atitudes sobre a tuberculose por estudantes universitários. Bol Campanha Nacional Contra Tuberculose 2004; 12(1):19-26.

13. Curley C. New guidelines: what to do about an unexpected positive tuberculin skin test. Clev Clin J Med 2000; 70(1):49-55

14. Breilh J. Epidemiologia economia, política e saúde. São Paulo: Hucitec; 1991.

15. Ayres JRCM. Sujeito, intersubjetividade e práticas de saúde. Rev C S Col 2001; 6(1):63-72.

Artigo apresentado em 28/01/2005

A provado em 26/06/2006

Versão final apresentada em 06/09/2006 